\title{
Особенности частотной зависимости проводимости неупорядоченных полупроводников в области перехода к режиму с постоянной длиной прыжка
}

\author{
(С) М.А. Ормонт, И.П. Звягин
}

Московский государственный университет им. М.В. Ломоносова (физический фракультет), 119991 Москва, Россия

E-mail: ormont@phys.msu.ru

(Получена 27 февраля 2017 г. Принята к печати 22 мая 2017 г.)

\begin{abstract}
Исследовано влияние эффектов гибридизации электронных состояний на высокочастотную проводимость неупорядоченных полупроводников. Показано, что зависимость предэкспоненциального множителя резонансного интеграла от межцентрового расстояния в паре обусловливает резкость смены механизмов проводимости в окрестности перехода частотной зависимости вещественной части проводимости $\sigma_{1}(\omega)$ от сублинейной к квадратичной. Резкость смены режимов проводимости связана с быстрым уменьшением длины прыжка с ростом частоты в окрестности кроссовера, что приводит к существенному относительному уменьшению вклада от бесфононной компоненты проводимости в режиме с переменной длиной прыжка с ростом частоты и переходу к режиму проводимости с постоянной длиной прыжка.
\end{abstract}

DOI: $10.21883 /$ FTP.2018.02.45437.8566

\section{1. Введение}

Как известно, диэлектрическая спектроскопия служит одним из методов получения информации об особенностях механизмов переноса носителей заряда и о локальной структуре материала [1,2]. Получение указанной информации связано с исследованием частотных зависимостей функций линейного отклика среды: комплексной диэлектрической восприимчивости $\alpha(\omega)=\alpha_{1}(\omega)+i \alpha_{2}(\omega)$, комплексной проницаемости $\varepsilon(\omega)=\varepsilon_{1}(\omega)+i \varepsilon_{2}(\omega)$ и комплексной проводимости $\sigma(\omega)=\sigma_{1}(\omega)+i \sigma_{2}(\omega)$.

Для многих неупорядоченных материалов (аморфные и легированные полупроводники, полупроводниковые стекла, проводящие полимеры, гранулированные проводники и т.п.) частотная зависимость вещественной части проводимости хорошо описывается степенной зависимостью [3]

$$
\sigma_{1}(\omega)=C \omega^{s}
$$

где $C, s$ - постоянные. Универсальность зависимости (1) затрудняет получение информации о конкретных особенностях механизма переноса. По этой причине исследования отклонений от универсальности и нахождение их связи со структурными особенностями материала и с особенностями переноса играют важную роль.

Как правило, в низкочастотной области имеем $0<s<1$, а с ростом частоты на кривых $\ln \sigma_{1}(\omega)$ от $\ln \omega$ наблюдается переход от сублинейного $(s \leq 1)$ к квадратичному $(s \approx 2)$ поведению (,излом“) [4-6].

Возрастающая частотная зависимость (1) указывает на прыжковый характер транспорта, причем такая зависимость обычно связывается с прыжками электронов по локализованным состояниям с участием фононов (релаксационная проводимость) [7]. Частотная зависимость вещественной части проводимости, близкая к линейной, получается при низких частотах и в теории низкотемпературной бесфононной (резонансной) прыжковой проводимости при учете кулоновских корреляций [8]. Теория бесфононной проводимости предсказывает переход (кроссовер) от линейной частотной зависимости $(s \approx 1[8])$ к зависимости, близкой к квадратичной $(s \approx 2[9])$, в области частот порядка $\omega_{\mathrm{cr}}$, при которых $\hbar \omega$ становится порядка энергии кулоновского взаимодействия между электронами внутри резонансных пар; при более низких частотах вещественная часть проводимости определяется фононным механизмом, а с ростом частоты бесфононная проводимость начинает преобладать над релаксационной. Низкотемпературные измерения (при температурах $T \sim 1 \mathrm{~K}$ ) частотной зависимости вещественной части проводимости $\sigma_{1}(\omega)$ в легированном кремнии $(\mathrm{Si}: \mathrm{P})$ на изоляторной стороне перехода металл-диэлектрик показали, что $\omega_{\mathrm{cr}} \sim 1$ ТГц [4-6].

Хотя теория качественно описывает общий характер частотной зависимости проводимости, существует ряд расхождений между теорией и экспериментальными данными. Согласно теории $[8,9]$, из-за гибридизации волновых функций изолированной пары центров и соответствующего ей отталкивания уровней наибольший вклад в бесфононную проводимость вносят пары центров, для которых межцентровые расстояния $r_{\lambda \lambda^{\prime}}$ удовлетворяют неравенствам $r_{\omega} \leq r_{\lambda \lambda^{\prime}} \leq r_{\omega}+a$, где $r_{\omega}=a \ln \left(2 I_{0} / \hbar \omega\right)$, $I_{0}=e^{2} / \kappa a, a-$ радиус локализации состояний, $\kappa$ - диэлектрическая проницаемость материала; при $r_{\lambda \lambda^{\prime}}<r_{\omega}$ отталкивание уровней становится $>\hbar \omega$, так что резонансные переходы невозможны. Характерная длина прыжка $r_{\omega}$ на частоте $\omega$ определяется из условия $\hbar \omega=2 I_{\lambda \lambda^{\prime}}\left(r_{\omega}\right)$, где $I_{\lambda \lambda^{\prime}}=I_{0} \exp \left(-r_{\lambda \lambda^{\prime}} / a\right)-$ резонансный интеграл, а $\lambda$ - номер центра. Проведенное в работе [10] рассмотрение показало, что в силу частотной зависимости характерной длины прыжка $r_{\omega}$ частотная зависимость $\sigma_{1}(\omega)$ может быть немонотонной, 
причем вплоть до частоты $\omega_{m}$, отвечающей максимуму $\sigma_{1}(\omega)$, кулоновское взаимодействие между электронами „активных“ (резонансных) пар играет основную роль $\hbar \omega<e^{2} / \kappa r_{\omega}$ и частотная зависимость $\sigma_{1}(\omega)$ остается близкой к линейной. Однако предсказываемая теорией немонотонность частотной зависимости бесфононной проводимости экспериментально не была обнаружена [4-6].

Наблюдаемая сублинейность $(s<1)$ частотной зависимости $\sigma_{1}(\omega)$ в области $\omega<\omega_{\text {cr }}$ может быть обусловлена частотной зависимостью характерной длины прыжка $r_{\omega}$ [7]. Отметим, что в области частот $\omega<\omega_{\mathrm{cr}}$ в некоторых работах наблюдалась суперлинейность $(s>1)$ [5,6]; это не согласуется с предсказываемой теорией сублинейностью $(s<1)$ (релаксационной [3] и бесфононной [8] компонент) в области промежуточных частот. В работах $[5,6]$ суперлинейность частотной зависимости низкотемпературной проводимости неупорядоченных полупроводников в области частот $\omega<\omega_{\mathrm{cr}}$ интерпретировалась как проявление кулоновской щели в одночастичной плотности состояний $[8,11]$. В $[12,13]$ было отмечено, что суперлинейность и монотонность экспериментально измеренных частотных зависимостей проводимости $\sigma_{1}(\omega)$ в переходной области частот могут быть связаны не с кулоновской щелью, а с переходом к режиму проводимости с постоянной длиной прыжка, когда основной вклад в проводимость вносят электронные переходы внутри пар с оптимальными межцентровыми расстояниями $r_{\text {opt }}$, слабо зависящими от частоты. Существование оптимального расстояния $r_{\mathrm{opt}}$ между центрами в парах обусловлено тем, что с уменьшением расстояния между центрами в паре уменьшается и изменение дипольного момента системы при электронном переходе, а с увеличением расстояния между центрами происходит экспоненциальное уменьшение перекрытия волновых функций состояний, отвечающих центрам локализации. Соответственно оптимальная длина прыжка $r_{\text {opt }} \approx 3 a$ определяется параметрами системы и слабо зависит от частоты. С ростом частоты характерная длина прыжка $r_{\omega}$ уменьшается. Переход к режиму проводимости с постоянной длиной прыжка $r_{\text {opt }}$ происходит в области частот, где $r_{\text {opt }}>r_{\omega}$; в этом случае основной вклад в проводимость вносят электронные переходы внутри пар с межцентровыми расстояниями $r_{\lambda \lambda^{\prime}} \sim r_{\text {opt}}$. Переход от линейной к квадратичной частотной зависимости вещественной части проводимости $\sigma_{1}(\omega)$ осушествляется в окрестности частоты, которая отвечает $r_{\omega} \sim r_{\text {opt }}[12,13]$.

\section{2. Влияние гибридизации электронных состояний на высокочастотную проводимость неупорядоченных полупроводников в переходной области}

Эффекты гибридизации волновых функций приводят к сдвигам энергий уровней, и учет этих эффектов заметно сказывается на частотной зависимости прыжковой проводимости $\sigma(\omega)$. В случае, когда расстояние между центрами в паре больше радиуса локализации, т. е. $r_{\lambda, \lambda^{\prime}}>a$, величины матричных элементов переноса можно непосредственно оценить. В приближении изотропного закона дисперсии имеем

$$
\begin{gathered}
s_{\lambda, \lambda^{\prime}} \approx\left(r_{\lambda, \lambda^{\prime}} / a\right)^{2} \exp \left(-r_{\lambda, \lambda^{\prime}} / a\right), \\
I_{\lambda, \lambda^{\prime}} \approx\left(e^{2} / \kappa a\right)\left(r_{\lambda, \lambda^{\prime}} / a\right) \exp \left(-r_{\lambda, \lambda^{\prime}} / a\right),
\end{gathered}
$$

где $\quad I_{\lambda, \lambda^{\prime}} \approx\left\langle\psi_{\lambda}\left|e^{2} / \kappa\right| \mathbf{r}-\mathbf{r}_{\lambda}|| \psi_{\lambda^{\prime}}\right\rangle, \quad \psi_{\lambda}=\left(1 / \sqrt{\pi a^{3}}\right)$ $\times \exp \left(-\left|\mathbf{r}-\mathbf{r}_{\lambda}\right| / a\right)$. Из выражений (2), (3) видно, что предэкспоненциальные множители резонансного интеграла и интеграла неортогональности в случае водородоподобных центров зависят от расстояния между центрами степенным образом. Действительно, при большом межцентровом расстоянии в паре, $r_{\lambda, \lambda^{\prime}} \gg a$, основной вклад в резонансный интеграл $I_{\lambda, \lambda^{\prime}}$ и интеграл неортогональности $s_{\lambda, \lambda^{\prime}}$ дает интегрирование по сигарообразной области, вытянутой вдоль отрезка $r_{\lambda, \lambda^{\prime}}$, внутри которой произведение волновых функций $\psi_{\lambda} \psi_{\lambda^{\prime}}$ можно считать постоянным и равным

$$
\psi_{\lambda} \psi_{\lambda^{\prime}} \approx\left(1 / a^{3}\right) \exp \left(-r_{\lambda, \lambda^{\prime}} / a\right) .
$$

Граница области, интегрирование по которой дает основной вклад в интегралы $I_{\lambda, \lambda^{\prime}}, s_{\lambda, \lambda^{\prime}}$, представляет собой поверхность, на которой значение произведения

$$
\psi_{\lambda} \psi_{\lambda^{\prime}} \approx\left(1 / a^{3}\right) \exp \left(-r_{\lambda, \lambda^{\prime}} / a\right) \exp \left[-\frac{1}{2} \frac{r_{\perp}^{2} r_{\lambda, \lambda^{\prime}}}{a\left(r_{\lambda, \lambda^{\prime}}-r_{\|}\right) r_{\|}}\right]
$$

уменьшается в $e$ раз по сравнению со значением на оси (4a); площадь поперечного сечения области порядка $S(\xi) \propto r_{\perp}^{2} \approx 2 a r_{\lambda, \lambda^{\prime}} \zeta(1-\zeta)$, где $\zeta=r_{\|} / r_{\lambda, \lambda^{\prime}}, \mathbf{r}_{\perp}, \mathbf{r}_{\|}-$ перпендикулярная и параллельная отрезку $r_{\lambda, \lambda^{\prime}}$ составляющие вектора $\mathbf{r}=\mathbf{r}_{\perp}+\mathbf{r}_{\|}, \xi \in[0,1]$ (начало отсчета совпадает с одним из центров, например $\lambda$ ). Оценка интеграла неортогональности (2) дает

$$
\begin{aligned}
s_{\lambda, \lambda^{\prime}} & =\left\langle\psi_{\lambda^{\prime}} \mid \psi_{\lambda}\right\rangle=\int_{V} \psi_{\lambda^{\prime}} \psi_{\lambda} d \mathbf{r} \approx \psi_{\lambda^{\prime}} \psi_{\lambda} \int_{V} d \mathbf{r} \\
& \approx a^{-3} \exp \left(-r_{\lambda, \lambda^{\prime}} / a\right) r_{\lambda, \lambda^{\prime}} \int_{0}^{1} S d \zeta \\
& \approx\left(\frac{r_{\lambda, \lambda^{\prime}}}{a}\right)^{2} \exp \left(-r_{\lambda, \lambda^{\prime}} / a\right)
\end{aligned}
$$

Полагая, что площадь $S$ порядка площади сферического сегмента, получаем оценку резонансного интеграла (3)

$$
\begin{aligned}
I_{\lambda, \lambda^{\prime}} & =\left\langle\psi_{\lambda}\left|U_{\lambda}\right| \psi_{\lambda^{\prime}}\right\rangle \approx \int_{V} \psi_{\lambda^{\prime}} \psi_{\lambda} \frac{e^{2}}{\kappa r_{\|}} S d r_{\|} \\
& \approx \frac{e^{2}}{\kappa} \psi_{\lambda^{\prime}} \psi_{\lambda} \int_{0}^{1} \frac{1}{\zeta} S d \varsigma \sim \frac{e^{2}}{\kappa a} \frac{r_{\lambda, \lambda^{\prime}}}{a} \exp \left(-r_{\lambda, \lambda^{\prime}} / a\right) .
\end{aligned}
$$


Степенная зависимость предэкспоненциальных множителей в интегралах $I_{\lambda, \lambda^{\prime}}, s_{\lambda, \lambda^{\prime}}$ от межцентрового расстояния $r_{\lambda, \lambda^{\prime}}$ определяется объемом сигарообразной области, увеличивающимся с ростом межцентрового расстояния. Учет этой зависимости приводит к увеличению длины прыжка $\tilde{r}_{\omega}>r_{\omega}$ и, как следствие, проводимости. Выражение для бесфононной проводимости в режиме с переменной длиной прыжка имеет вид [8]

$$
\sigma_{1}^{\mathrm{res}}(\omega)=\frac{\pi^{2} e^{2} \rho_{0}^{2} a}{3} r_{\omega}^{4} \omega\left(\hbar \omega+\frac{e^{2}}{\kappa r_{\omega}}\right)
$$

где $I_{\lambda, \lambda^{\prime}} \approx\left(e^{2} / \kappa a\right) \exp \left(-r_{\lambda, \lambda^{\prime}} / a\right), \hbar \omega=2 I_{\lambda, \lambda^{\prime}}\left(r_{\omega}\right)$.

Соотношение (5a) удобно представить в виде

$$
\sigma_{1}^{\mathrm{res}}(\omega)=\sigma_{0}\left[\ln \left(\frac{\omega_{c}}{\omega}\right)\right]^{4} \frac{\omega}{\omega_{c}}\left[A^{-1} \frac{\omega}{\omega_{c}}+\frac{1}{\ln \left(\omega_{c} / \omega\right)}\right],
$$

где $\sigma_{0}=\pi^{2} e^{4} \rho_{0}^{2} a^{4} \omega_{c} / 3 \kappa, \quad \omega_{c}=2 I_{0} / \hbar, \quad I_{0}=e^{2} / \kappa a$, $A=e^{2} /\left(\kappa a \hbar \omega_{c}\right)=e^{2} /\left(2 I_{0} \kappa a\right) \approx 1 / 2$.

При учете степенно́й зависимости предэкспоненциального множителя резонансного интеграла $\tilde{I}_{\lambda \lambda^{\prime}}$ от межцентрового расстояния выражение для бесфононного вклада в проводимость имеет вид

$$
\tilde{\sigma}_{1}^{\mathrm{res}}(\omega)=\frac{\pi^{2} e^{2} \rho_{0}^{2} a}{3} \tilde{r}_{\omega}^{4} \omega\left(\hbar \omega+\frac{e^{2}}{\kappa \tilde{r}_{\omega}}\right)
$$

где

$$
\tilde{I}_{\lambda, \lambda^{\prime}} \approx \frac{e^{2}}{\kappa a} \frac{r_{\lambda, \lambda^{\prime}}}{a} \exp \left(-r_{\lambda, \lambda^{\prime}} / a\right),
$$

a длина прыжка $\tilde{r}_{\omega}$ на частоте $\omega$ (с учетом предэкспоненциального множителя) определяется из условия $\hbar \omega=2 \tilde{I}_{\lambda, \lambda^{\prime}}\left(\tilde{r}_{\omega}\right)$. Равенства $(5 \mathrm{a}),(56),(6)$ справедливы в области частот $\omega<\omega_{c}$, соответствующих неравенствам $r_{\omega}, \tilde{r}_{\omega}>a$; при этом с ростом частоты $\omega$ длина $\tilde{r}_{\omega}$ уменьшается. Пары с межцентровыми расстояниями $r_{\lambda, \lambda^{\prime}}<r_{\omega}, \tilde{r}_{\omega}$ вклада в проводимость не дают, поскольку отталкивание уровней становится бо́льшим $\hbar \omega$ и переходы невозможны.

Из равенства $I_{\lambda, \lambda^{\prime}}\left(r_{\omega}\right)=\tilde{I}_{\lambda, \lambda^{\prime}}\left(\tilde{r}_{\omega}\right)$ следует, что длины $r_{\omega}$ и $\tilde{r}_{\omega}$ связаны соотношением

$$
\frac{\tilde{r}_{\omega}}{a}-\ln \left(\frac{\tilde{r}_{\omega}}{a}\right)=\frac{r_{\omega}}{a} .
$$

Поскольку $r_{\omega} / a=\ln \left(\omega_{c} / \omega\right)$, соотношение (7) определяет частотную зависимость бесфононной проводимости $\tilde{\sigma}_{1}^{\text {res }}\left(\omega / \omega_{c}\right)$. Согласно $(7)$ с ростом частоты характерные длины переходов $r_{\omega}$ и $\tilde{r}_{\omega}$ уменьшаются; при этом $\tilde{r}_{\omega}>r_{\omega}$ (рис. 1).

На рис. 2, а приведена зависимость от частоты бесфононного вклада в проводимость $\tilde{\sigma}_{1}^{\text {res }}\left(\omega / \omega_{c}\right)$ с учетом зависимости предэкспоненциального множителя резонансного интеграла $\tilde{I}_{\lambda, \lambda^{\prime}}$ от расстояния между центрами $r_{\lambda, \lambda^{\prime}}$ (рис. 2, $a$, кривая 2). Проводимость нормирована на величину $\sigma_{0}=\pi^{2} e^{4} a^{4} \rho_{0}^{2} \omega_{c} / 3 \kappa$; например, для случая $\mathrm{Si}: \mathrm{P}$ при $a \approx 30 \AA, \rho_{0} \sim 10^{18} \mathrm{~cm}^{-3} \cdot{ }^{-} \mathrm{B}^{-1}, \kappa \approx 12$, $\omega_{c} \sim 10^{13}$ рад $\cdot \mathrm{c}^{-1}$ имеем $\sigma_{0} \approx 0.007 \mathrm{OM}^{-1} \cdot \mathrm{cm}^{-1}$. Для

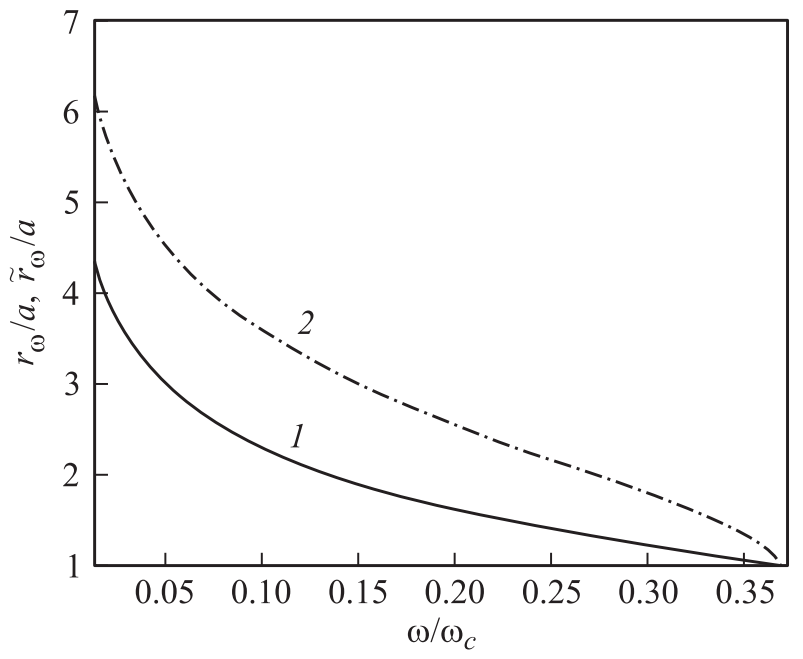

Рис. 1. Зависимости длин $r_{\omega}(1), \tilde{r}_{\omega}(2)$ от частоты.

сравнения на рис. 2, а приведена частотная зависимость бесфононного вклада в проводимость в случае с постоянным предэкспоненциальным множителем резонансного интеграла $I_{\lambda, \lambda^{\prime}}$ (кривая 1). Из рис. 3 видно, что частотная зависимость бесфононной проводимости (6) с учетом степенной зависимости предэкспоненты резонансного интеграла от межцентрового расстояния в паре в низкочастотной области (при $\left.\hbar \omega<e^{2} / \kappa r_{\omega}\right)$ сохраняет сублинейный характер: $\tilde{\sigma}_{1}^{\mathrm{res}}(\omega) \propto \omega^{s}$, где $s \approx 0.8$. Приведенные зависимости $\sigma_{1}(\omega)$ (кривые 1,2 на рис. 2,a) немонотонны и имеют максимум в окрестности частоты $\omega_{m} \approx 0.1 \omega_{c}$ (кривая 2), $\omega_{m} \approx 0.07 \omega_{c}$ (кривая 1$)$. Немонотонность $\sigma_{1}(\omega)$ обусловлена частотной зависимостью оптимальных длин прыжка $r_{\omega}, \tilde{r}_{\omega}$, причем почти вПлоть до частоты $\omega_{m}$, отвечающей максимуму $\sigma_{1}(\omega)$, кулоновское взаимодействие между электронами „активных“ пар играет основную роль и частотные зависимости $\sigma_{1}(\omega)$ остаются близкими к линейной (рис. 3 ).

Отметим, что вид частотной зависимости проводимости (5б) зависит от безразмерного параметра $A$. Согласно $[10]$, переход от линейной к квадратичной частотной зависимости проводимости $\sigma_{1}^{\text {res }}(\omega)$ в режиме с переменной длиной прыжка $r_{\omega}$ может наблюдаться лишь при достаточно малых значениях параметра, $A<10^{-5}$, когда $\omega_{\mathrm{cr}}<\omega_{m}$; при этом для водородоподобных центров параметр $A$ порядка единицы $(A=0.5)$ и имеем $\hbar \omega<e^{2} / \kappa r_{\omega}$ вплоть до перехода на падающий участок кривой $\sigma_{1}^{\mathrm{res}}(\omega)$ (рис. 3). Максимум $\sigma_{1}^{\text {res }}(\omega)$ достигается в окрестности частоты $\omega_{m} \approx \exp (-3) \omega_{c} \approx 0.05 \omega_{c}$; при этом положение максимума $\omega_{m}$ слабо зависит от параметра $A$. Такое поведение частотной зависимости проводимости в зависимости от значений параметра $A$ сохраняется и для $\tilde{\sigma}_{1}^{\text {res }}(\omega)$, т. е. при учете зависимости предэкспоненциального множителя резонансного интеграла $\tilde{I}_{\lambda, \lambda^{\prime}}$ от межцентрового расстояния в паре. Отметим, однако, что предсказываемая стандартной теорией немонотонность $\sigma_{1}(\omega)$ вблизи области кроссовера не наблюдается [4-6]. Соответственно получаемые в стандартной теории ча- 

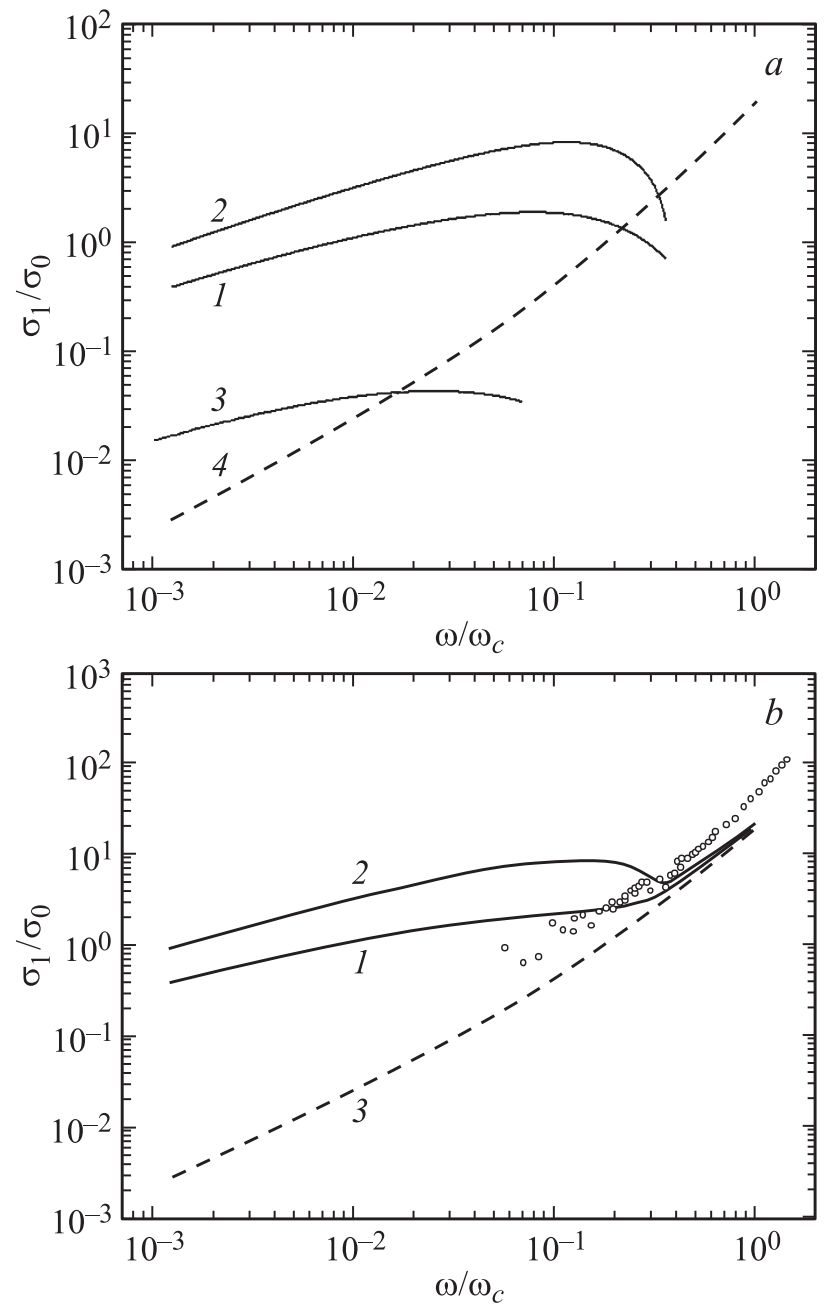

Рис. 2. Частотные зависимости вещественной части проводимости $\sigma_{1} / \sigma_{0} . a: 1-$ подход на основе теории бесфононной проводимости в режиме с переменной длиной прыжка $r_{\omega}(5 \mathrm{a})$, (5б) без учета зависимости предэкспоненциального множителя резонансного интеграла от межцентрового расстояния в паре, т.е. с постоянным предэкспоненциальным множителем; 2 - подход на основе теории бесфононной проводимости в режиме с переменной длиной прыжка $\tilde{r}_{\omega}$ (6) с учетом зависимости предэкспоненциального множителя резонансного интеграла от межцентрового расстояния в паре; 3 релаксационная проводимость (8a), (8б) с переменной длиной прыжка $\bar{r}_{\omega} ; 4-$ проводимость в режиме с постоянной длиной прыжка (11). $b$ : 1,2 - экстраполяции моделей с переменной длиной прыжка (соответственно с постоянной предэкспонентой резонансного интеграла и с предэкспонентой, зависящей от межцентрового расстояния) на область квадратичной частотной зависимости в режиме проводимости с постоянной длиной прыжка; точки - экспериментальные данные [5]. 3 - проводимость в режиме с постоянной длиной прыжка (11).

стотные зависимости проводимости в режиме с переменной длиной прыжка $r_{\omega}$ при типичных для мелких примесей значениях параметров $(A \approx 0.5)$ не описывают наблюдаемый переход частотной зависимости прово- димости $\sigma_{1}(\omega)$ неупорядоченных полупроводников от почти линейной к квадратичной с ростом частоты.

Отметим, что выражения для вещественной части бесфононной проводимости в низкочастотном режиме (второе слагаемое в (5a)) и для низкотемпературной релаксационной проводимости - одного вида. На рис. 2, $a$ для сравнения приведена частотная зависимость низкотемпературной релаксационной проводимости (кривая 3) [14]

$$
\sigma_{1}^{\mathrm{rel}}=\frac{\pi^{2} e^{4} \rho_{0}^{2} a}{6 \kappa} \omega \bar{r}_{\omega}^{3}
$$

Здесь $\bar{r}_{\omega}=(a / 2) \ln \left(\omega_{\mathrm{ph}} / \omega\right)$ - характерная длина прыжка при релаксационной проводимости. Выражение (8a) удобно представить в виде

$$
\sigma_{1}^{\mathrm{rel}}=\sigma_{0} \frac{1}{16} \frac{\omega}{\omega_{c}}\left[\ln \left(B \frac{\omega_{c}}{\omega}\right)\right]^{3},
$$

где $B=\omega_{\mathrm{ph}} / \omega_{c} \approx 1$. Отметим, что при типичных для мелких примесей значениях параметра $A \approx 0.5$ суперпозиция релаксационного вклада в проводимости $\sigma_{1}^{\text {rel }}$ и бесфононного вклада $\sigma_{1}^{\text {res,Mott }}(\omega)$ [9],

$$
\begin{gathered}
\sigma_{1}^{\mathrm{res}, \operatorname{Mott}}(\omega)=\frac{\pi^{2} e^{2} \rho_{0}^{2} a \hbar}{3} r_{\omega}^{4} \omega^{2}, \\
\sigma_{1}^{\mathrm{res}, \operatorname{Mott}}(\omega)=\sigma_{0}\left[\ln \left(\frac{\omega_{c}}{\omega}\right)\right]^{4} A^{-1}\left(\frac{\omega}{\omega_{c}}\right)^{2},
\end{gathered}
$$

не описывает перехода частотной зависимости проводимости от линейной к квадратичной с ростом часто-

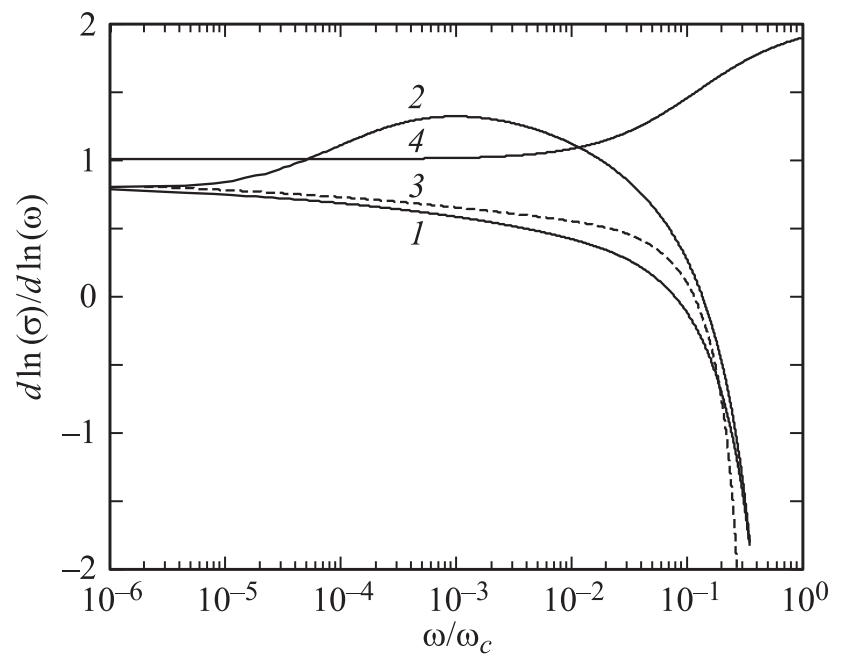

Рис. 3. Частотные зависимости $s\left(\omega / \omega_{c}\right)=d \ln (\sigma) / d \ln (\omega)$ $=\frac{\omega / \omega_{c}}{\sigma / \sigma_{0}} \frac{d \sigma / \sigma_{0}}{d \omega / \omega_{c}} \cdot 1-$ случай бесфононной проводимости $(5 \mathrm{a})$, (5б) с переменной длиной прыжка, 2 отвечает значению безразмерного параметра $A=0.001$ в (5б); 3 - случай бесфононной проводимости в режиме с переменной длиной прыжка с учетом зависимости предэкспоненты резонансного интеграла от межцентрового расстояния в паре $(A=1 / 2) ; 4-$ случай бесфононной проводимости в режиме с постоянной длиной прыжка, 4 отвечает режиму с постоянной длиной прыжка $r_{\mathrm{opt}}$ во всей области перехода от линейной к квадратичной частотной зависимости проводимости. 
ты. Действительно, выражение для вещественной части бесфононной проводимости в низкочастотном режиме (второе слагаемое в (5a)) имеет вид [8]

$$
\begin{gathered}
\sigma_{1}^{\mathrm{res}, \mathrm{Ef}-\mathrm{Sh}}(\omega)=\frac{\pi^{2} e^{4} \rho_{0}^{2} a}{3 \kappa} r_{\omega}^{3} \omega, \\
\sigma_{1}^{\mathrm{res}, \mathrm{Ef}-\mathrm{Sh}}(\omega)=\sigma_{0}\left[\ln \left(\frac{\omega_{c}}{\omega}\right)\right]^{3} \frac{\omega}{\omega_{c}} ;
\end{gathered}
$$

при этом отношение вещественных частей релаксационной проводимости $\sigma_{1}^{\text {rel }}(8 б)$ и бесфононной проводимости $\sigma_{1}^{\text {res,Ef-Sh }}(10 б)$ при $B=1$ постоянно и равно $\sigma_{1}^{\text {rel }} / \sigma_{1}^{\text {res, Ef }-\mathrm{Sh}}=1 / 16$. Согласно (8б) и (9б) кроссовер от линейной (релаксационной) к квадратичной (бесфононной) частотной зависимости проводимости $\sigma_{1}(\omega)$ в режиме с переменной длиной прыжка может наблюдаться лишь при достаточно малых значениях параметра, $A<10^{-5}$; при этом за счет частотной зависимости оптимальной длины прыжка $r_{\omega}$ частотная зависимость проводимости $\sigma_{1}(\omega)$ будет немонотонна с максимумом в окрестности частоты перехода.

В области частот, когда $r_{\text {opt }}>r_{\omega}$, эффекты, связанные с гибридизацией локализованных состояний, становятся несущественны; при этом происходит переход от проводимости в режиме с переменной длиной прыжка к бесфононной проводимости в режиме с постоянной длиной прыжка. Соответственно в режиме с постоянной длиной прыжка имеем [12]

$$
\sigma_{1}^{\mathrm{res}}(\omega)=\frac{\pi^{2}}{3} C_{1} e^{2} \rho_{0}^{2} a^{5} \omega\left(\hbar \omega+C_{2} \frac{e^{2}}{\kappa a}\right),
$$

где $C_{1}, C_{2}$ - численные коэффициенты $\left(C_{1}=8.75\right.$, $\left.C_{2}=1 / 4\right)$. На рис. 2, $a$ этому случаю соответствует кривая 4. Выражение (11a) удобно представить в виде

$$
\sigma_{1}^{\mathrm{res}}(\omega)=\sigma_{0} C_{1} \frac{\omega}{\omega_{c}}\left(A^{-1} \frac{\omega}{\omega_{c}}+C_{2}\right) .
$$

C ростом частоты характерная длина прыжка $r_{\omega}$ уменьшается, и в области высоких частот происходит переход к режиму проводимости с постоянной длиной прыжка (11б), когда основной вклад в проводимость вносят электронные переходы внутри пар с оптимальными межцентровыми расстояниями $r_{\mathrm{opt}} \approx 3 a$, слабо зависящими от частоты. Переход к режиму проводимости с постоянной длиной прыжка $r_{\text {opt }}$ происходит в области частот $\omega>\exp (-3) \omega_{c} \approx 0.05 \omega_{c}$, когда $r_{\text {opt }}>r_{\omega}$; в этом случае основной вклад в проводимость вносят электронные переходы внутри пар с межцентровыми расстояниями $r_{\lambda \lambda^{\prime}} \sim r_{\text {орt. }}$ Отметим, что частота $\omega \approx 0.05 \omega_{c}$, при которой происходит указанный выше переход, порядка частоты кроссовера в (11б), $\hbar \omega=C_{2} e^{2} / \kappa a \approx e^{2} / \kappa r_{\text {opt }}$, т.е. $\omega_{\mathrm{cr}} \approx 0.1 \omega_{c}$. Соответственно переход от линейной к квадратичной частотной зависимости вещественной части проводимости осуществляется в окрестности частоты $\omega \approx 0.05 \omega_{c}$, которая отвечает $r_{\omega} \sim r_{\text {opt }}[12]$.

Экстраполяция результата стандартного подхода из области линейной частотной зависимости в режиме с переменной длиной прыжка на область квадратичной частотной зависимости проводимости в режиме с постоянной длиной прыжка представлена кривыми 1,2 на рис. $2, b$.

С ростом частоты $\omega$ длина прыжка $\tilde{r}_{\omega}$ уменьшается и по мере приближения к частоте кроссовера $\omega_{\text {cr }}$ стремится к величине $r_{\omega}$ (рис. 1). Учет степенной зависимости предэкспоненциального множителя резонансного интеграла $I_{\lambda, \lambda^{\prime}}$ от расстояния между центрами в режиме с переменной длиной прыжка приводит к существенному относительному уменьшению вклада от бесфононной компоненты проводимости (6) в режиме с переменной длиной прыжка с ростом частоты и обусловливает резкость перехода к режиму проводимости с постоянной длиной прыжка в области $r_{\omega} \approx r_{\mathrm{opt}}$ (рис. 2). Такое поведение частотной зависимости проводимости $\tilde{\sigma}_{1}^{\mathrm{res}}(\omega)$ обусловлено увеличением длины прыжка $\tilde{r}_{\omega}>r_{\omega}$ и соответственно величины проводимости и связано с быстрым уменьшением длины прыжка $\tilde{r}_{\omega}$ с ростом частоты в области перехода. Соответственно резкость смены механизмов проводимости связана с быстрым уменьшением длины прыжка $\tilde{r}_{\omega}$ с ростом частоты в окрестности кроссовера.

Отметим, что в рассматриваемом случае мелких примесных уровней вдали от области перехода, т.е. при $\omega \ll \omega_{\text {cr }}$ и $\omega>\omega_{\text {cr }}$, эффекты гибридизации электронных состояний несущественны. В области частот $\omega \ll \omega_{c r}$ резонансный интеграл мал, $I_{\lambda, \lambda,}\left(\bar{r}_{\omega}\right)<k T ; \bar{r}_{\omega}=(a / 2) \ln \left(\omega_{\mathrm{ph}} / \omega\right)$ - характерная длина прыжка при релаксационной проводимости, где $\omega_{\mathrm{ph}} \sim 10^{13}$ рад/с. Вещественная часть проводимости определяется релаксационной компонентой, $\quad \sigma_{1}(\omega) \approx \sigma_{1}^{\text {rel }}(\omega), \quad \sigma_{1}^{\text {rel }}=\left(\pi^{4} e^{2} \rho_{0}^{2} a / 24\right) \omega k T \bar{r}_{\omega}^{4}$ при $U\left(\bar{r}_{\omega}\right)=e^{2} / \kappa \bar{r}_{\omega}<k T[15]$ и $\sigma_{1}^{\text {rel }}=\left(\pi^{2} e^{4} \rho_{0}^{2} a / 6 \kappa\right) \omega \bar{r}_{\omega}^{3}$ при низких температурах, $U\left(\bar{r}_{\omega}\right)=e^{2} / \kappa \bar{r}_{\omega} \gg k T$ [14].

С ростом частоты (т.е. с уменьшением длины прыжка $\left.\bar{r}_{\omega}\right)$ бесфононная проводимость начинает преобладать над релаксационной, т.е. $\sigma_{1}(\omega) \approx \sigma_{1}^{\text {res }}(\omega)$. При частотах $\omega>\omega_{\text {cr }}$, отвечающих $\tilde{r}_{\omega}<r_{\text {opt }} \approx 3 a$, происходит подавление гибридизации и основной вклад в проводимость вносят переходы внутри пар центров с межцентровым расстоянием порядка $r_{\text {opt }}$, не зависящим от частоты [12,13].

\section{3. Заключение}

Таким образом, получаемые в стандартной теории частотные зависимости проводимости в режиме с переменной длиной прыжка $r_{\omega}$ при типичных значениях параметров $(A \sim 1)$ не описывают наблюдаемый переход частотной зависимости проводимости $\sigma_{1}(\omega)$ неупорядоченных полупроводников от сублинейной к суперлинейной с ростом частоты. Получавшаяся в расчете немонотонность частотной зависимости проводимости $\sigma_{1}^{\text {res }}(\omega)$ в режиме с переменной длиной прыжка связана с частотной зависимостью характерной длины прыжка, причем почти вплоть до частоты $\omega_{m}$, отвечающей 
максимуму $\sigma_{1}^{\text {res }}(\omega)$, кулоновское взаимодействие между электронами „активных“ пар играет основную роль и частотная зависимость $\sigma_{1}(\omega)$ при $\omega<\omega_{m}$ остается близкой к линейной.

Переход к суперлинейной частотной зависимости проводимости $\sigma_{1}(\omega)$ может быть связан с переходом к режиму проводимости с постоянной длиной прыжка, когда основной вклад в проводимость вносят электронные переходы внутри пар с оптимальными межцентровыми расстояниями $r_{\text {орt }}$, слабо зависящими от частоты. Учет степенной зависимости предэкспоненциального множителя резонансного интеграла $\tilde{I}_{\lambda \lambda^{\prime}}$ от расстояния между центрами в паре приводит к существенному относительному уменьшению вклада от бесфононной компоненты проводимости в режиме с переменной длиной прыжка в области перехода к режиму проводимости с постоянной длиной прыжка. Такое поведение частотной зависимости проводимости $\tilde{\sigma}_{1}^{\text {res }}(\omega)$ обусловлено увеличением длины прыжка $\tilde{r}_{\omega}>r_{\omega}$, соответственно величины проводимости и связано с быстрым уменьшением длины прыжка $\tilde{r}_{\omega}$ с ростом частоты в области перехода.

Таким образом, характер кривых частотной зависимости проводимости $\sigma_{1}(\omega)$ в области „излома“ указывает на изменение режима проводимости (переход от переменной к постоянной длине прыжка с ростом частоты); при этом резкость смены режимов бесфононной проводимости связана с зависимостью предэкспоненциального множителя резонансного интеграла $\tilde{I}_{\lambda \lambda^{\prime}}$ от межцентрового расстояния в паре.

\section{Список литературы}

[1] F. Kremer, A. Schonhals. Broadband dielectric spectroscopy (Berlin-N.Y., Springer, 2003).

[2] Г. Фрелих. Теория диэлектриков. Диэлектрическая проницаемость и диэлектрические потери (пер. с англ.) (М., ИЛ, 1960).

[3] I.P. Zvyagin. In: Charge Transport in Disordered Solids with Applications in Electronics, ed. by S. Baranovski (John Wiley \& Sons, Chichester, 2006) p. 339.

[4] E. Helgren, N.P. Armitage, G. Gruner. Phys. Rev. B, 69, 014201 (2004).

[5] M. Hering, M. Scheffler, M. Dressel, H.v. Lohneysen. Phys. Rev. B, 75, 205203 (2007).

[6] E. Ritz, M. Dressel. Phys. Status Solidi C, 5, 703 (2008).

[7] M. Pollak, T.H. Geballe. Phys. Rev., 122, 1742 (1961).

[8] Б.И. Шкловский, А.Л. Эфрос. ЖЭТФ, 81, 406 (1981).

[9] N.F. Mott. Phil. Mag., 22, 7 (1970).

[10] И.П. Звягин, М.А. Ормонт. Вестн. Моск. ун-та. Физика, Астрономия, № 4, 44 (2008).

[11] Б.И. Шкловский, А.Л. Эфрос. Электронные свойства легированных полупроводников (М., Наука, 1979).

[12] М.А. Ормонт, И.П. Звягин. ФТП, 49 (4), 449 (2015).

[13] М.А. Ормонт. ФТП, 49 (10), 1314 (2015).

[14] A.L. Efros. Phil. Mag. B, 43, 829 (1981).

[15] I.G. Austin, N.F. Mott. Adv. Phys., 18, 41 (1969).

\section{Frequency dependence of disordered semiconductor conductivity in the region of the crossover to the fixed-range hopping regime}

\author{
M.A. Ormont, I.P. Zvyagin \\ Lomonosov Moscow State University (Physics Faculty), \\ 119991 Moscow, Russia
}

\begin{abstract}
We study the effect of hybridization of electron states on high-frequency resonance conductivity of disordered semiconductors corresponding to electron transitions to distant centers. It is shown that the dependence of the pre-exponential factor of the resonance integral on the inter-center separation in pair determines sharpness of the transition between the conduction regimes near the crossover from sublinear to superlinear frequency dependence of the real part of conductivity. The sharpness of the transition between the conduction regimes is associated with a decrease in the hopping distance with increasing frequency near the crossover which gives rise to a significant reduction of the contribution from phononless conductivity in the variable-range hopping regime and transition to fixed-range hopping regime.
\end{abstract}

